\title{
Letter legibility and visual word recognition
}

\author{
TATJANA A. NAZIR \\ Center for Research in Cognitive Neuroscience, CNRS, Marseille, France \\ ARTHUR M. JACOBS \\ Philipps-Universität, Marburg, Germany, \\ and Center for Research in Cognitive Neuroscience, CNRS, Marseille, France \\ and \\ J. KEVIN O'REGAN \\ Laboratoire de Psychologie Expérimentale, CNRS, Paris, France
}

\begin{abstract}
Word recognition performance varies systematically as a function of where the eyes fixate in the word. Performance is maximal with the eye slightly left of the center of the word and decreases drastically to both sides of this optimal viewing position. While manipulations of lexical factors have only marginal effects on this phenomenon, previous studies have pointed to a relation between the viewing position effect (VPE) and letter legibility: When letter legibility drops, the VPE becomes more exaggerated. To further investigate this phenomenon, we improved letter legibility by magnifying letter size in a way that was proportional to the distance from fixation (e.g., TABLE). Contrary to what would be expected if the VPE were due to limits of acuity, improving the legibility of letters has only a restricted influence on performance. In particular, for long words, a strong VPE remains even when letter legibility is equalized across eccentricities. The failure to neutralize the VPE is interpreted in terms of perceptual learning: Since normally, because of acuity limitations, the only information available in parafoveal vision concerns low-resolution features of letters; even when magnification provides better information, readers are unable to make use of it.
\end{abstract}

The legibility of a letter is known to decrease with its distance from the position where the eye is fixating (Anstis, 1974; Bouma, 1970; Nazir, Heller, \& Sussmann, 1992; Nazir, O'Regan, \& Jacobs, 1991; Olzak \& Thomas, 1986; Townsend, Taylor, \& Brown, 1971). Even for unlimited presentation durations, the probability of identifying a letter in a random string drops from a value of 1 for the directly fixated letter to approximately 0.4 for a letter presented only five letter locations away from the center of gaze (Townsend et al., 1971). Given that, during normal reading, many words are fixated only once and that the average fixation duration is less than $250 \mathrm{msec}$ (see, e.g., Rayner \& Pollatsek, 1989), these limits of acuity must necessarily affect word recognition performance: We expect a word to be recognized best when the eye is fixating its

This research was supported in part by grants from the Deutsche Forschungsgemeinschaft; Mittel der Forschergruppe "Dynamik und hierarchisch-parallele Strukturierung von Repräsentationen in Nervensystemen" an der Philipps-Universität, Marburg, to T.A.N. and A.M.J. The authors thank P. Marshal for software assistance. We are grateful to W. Estes, G. Loftus, D. Massaro, M. Montant, H. C. Nürk, and an anonymous reviewer for helpful comments on this work. Correspondence concerning this article should be addressed to T. A. Nazir, Center for Research in Cognitive Neuroscience (CNRS-CRNC), 31, Chemin Joseph-Aiguier 13402 Marseille Cedex 9, France (e-mail: nazir@lnf.cnrs-mrs.fr).

-Accepted by previous editor, Geoffrey R. Loftus center, where the largest number of letters benefits from high resolution.

Eye movement behavior during normal reading seems to confirm this hypothesis. The probability that a word is fixated more than once is minimal when the eye lands near the middle of a word and increases as the eye's initial fixation position deviates either leftward or rightward from this optimal viewing position (McConkie, Kerr, Reddix, Zola, \& Jacobs, 1989; O'Regan, Lévy-Schoen, Pynte, \& Brugaillère, 1984; Radach \& Kempe, 1993; Underwood, Clews, \& Everatt, 1990; Vitu, 1991b; Vitu, O'Regan, \& Mittau, 1990). Under the assumption that refixations result from the failure to identify the word during the previous fixation, this behavior is compatible with the hypothesis that the amount of available visual information differs significantly as a function of the eye's first location in the word. The existence of a strong viewing position effect (VPE) during word recognition has also been confirmed with dependent measures other than eye movements. Naming latencies and lexical decision times (when the eye is free to move in the word) are shortest when the eye starts to fixate the word near its center (Brysbaert \& d'Ydewalle, 1988; O'Regan \& Jacobs, 1992; O'Regan et al., 1984), and the probability of correct lexical decision and correct identification (when only one fixation is allowed in the word) is higher when the word is fixated near the center than when the word is fixated toward the beginning or the end (see, e.g., Brysbaert, Vitu, \& Schroyens, 
1996; Farid \& Grainger, 1996; Nazir, 1993; Nazir et al., 1992; Nazir et al., 1991).

\section{Characteristics of the Viewing Position Effect}

A typical pattern of the VPE for five- to nine-letter words is shown in Figure 1 (solid symbols; data replotted from Nazir, 1993). The data were collected in a lexical decision task where subjects were asked to decide whether a briefly presented letter string was a word or not. The experimental technique consisted of presenting the letter string laterally displaced in relation to a fixation point, such that, on its appearance, the eye was fixating one of five fixation zones in the word. (Each stimulus string was divided into five equally wide zones of one fifth of the width of the total word length. Thus, the zones for a five-letter string were one-letter wide; for a 9-letter string, they were 1.8 letters wide. The center of each zone was designated as a potential initial fixation point.) As can be seen from the figure, performance for all word lengths varied systematically with the location of the eye in the word. Overall performance-indicated in the right upper corner of each panel-dropped, on average, $3.3 \%$ with each additional letter of word length, and the VPE became stronger for longer words. Note, however, that all of the curves were asymmetric. The optimal viewing position was systematically left of and not at word center.

At first glance, this asymmetry contradicts the hypothesis of a direct relation between the VPE and letter legibility. Since acuity drops symmetrically to both sides of fixation (Olzak \& Thomas, 1986), symmetrical curves are expected. O'Regan and Jacobs (1992) hypothesized that this shift of the optimal viewing position away from the center of the word is related to the informational and/or morphological structure of words. If the first half of words tends to be more informative than the second half (in the sense that only a few other words share the same beginning with a given target word), there might be an advantage in fixating left of the center of a word.
However, for this hypothesis to be correct, the optimal viewing position should move into the second half of words when the word stimuli are chosen to be more informative at their ends (e.g., for words like interview, which shares its prefix with many other words). O'Regan et al. (1984; see, as well, Brysbaert et al., 1996, and Holmes \& O'Regan, 1987) showed some empirical evidence in favor of such a dependence of the VPE on the informational/morphological structure of words. However, the observed dependence was very small, and furthermore, in none of the reported experiments did the optimal viewing position shift rightward beyond the center of the word.

Other investigators, such as Brysbaert and d'Ydewalle (1988), suggested that the leftward shift of the optimal viewing position is related to hemispheric differences in language processing. To test this hypothesis, the authors measured word recognition as a function of viewing position for left- and right-hemisphere dominant subjects. Although right-hemisphere dominant subjects performed slightly differently from left-dominant subjects, none of the right-dominant subjects ever showed an optimal viewing position in the second half of the word. Thus, as was the case for the informational structure of words, hemispheric differences in language processing do not explain the strong and robust asymmetry in the VPE.

As an alternative, Nazir et al. (1991) proposed that the observed asymmetry is caused by differences in the visibility of letters on the two sides of the fixation point. The probability of recognizing letters, especially when they are embedded in strings, is higher when the letters are presented in the right than in the left visual field (see, e.g., Bouma, 1973; Bouma \& Legein, 1977; Hagenzieker, van der Heijden, \& Hagenaar, 1990; Nazir et al., 1991). According to an estimation by Nazir et al., the ratio of this left/right asymmetry in the legibility of letter is approximately $1.8 / 1$. Thus, when the probability of recognizing a target letter drops from 1 at the center of gaze to .9 at a given eccentricity in the right visual field, it drops
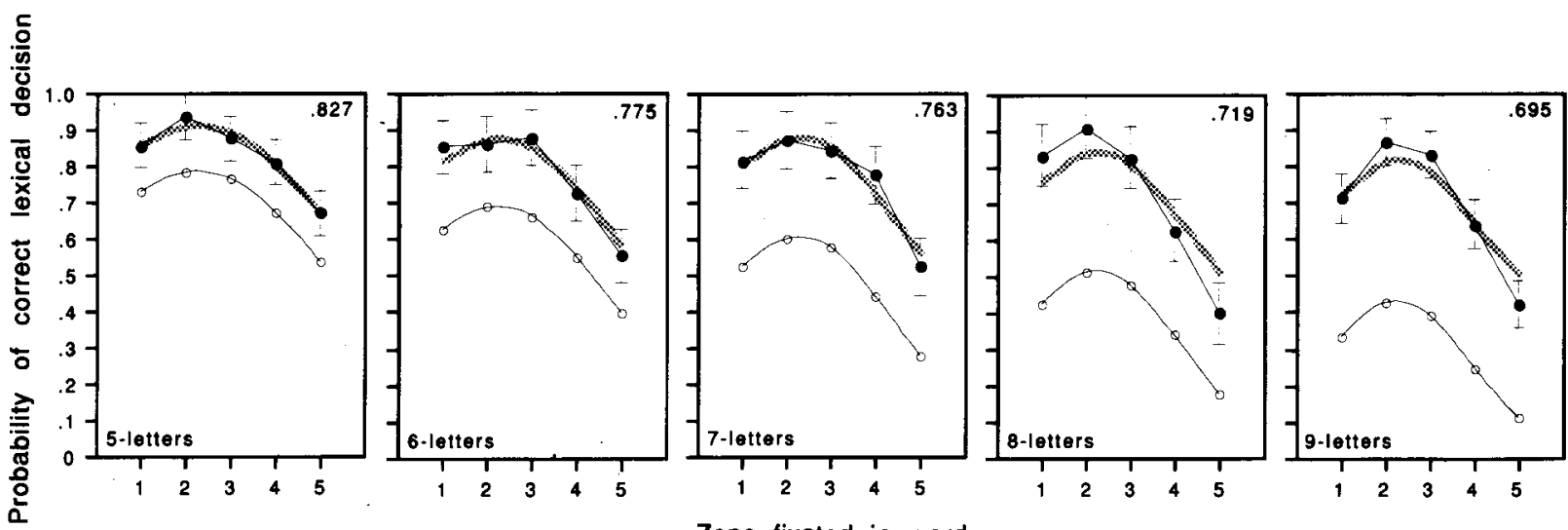

Zone fixated in word

Figure 1. The probability of correct lexical decision for five- to nine-letter words as a function of the zone fixated in the word (solid symbols; data replotted from Nazir, 1993), plotted with a 95\% confidence interval (Loftus \& Masson, 1994). The curves with open symbols represent theoretical data (see text). The gray curves represent the same theoretical data shifted in height to match the empirical data. The values in the upper right corner indicate observed performance averaged over all fixation zones. 
to .82 when the letter is presented at the same eccentricity in the left visual field. When a word has to be recognized, it is, therefore, advantageous to fixate to the left of the center of the word so that fewer letters have to be identified in the left visual field. A simple mathematical model, proposed by Nazir et al. (1991), illustrates this idea (see also McConkie et al., 1989, for a similar model predicting refixation probabilities).

\section{A Model to Account for the VPE}

In the model by Nazir et al. (1991), it is assumed that (1) word recognition is letter based (see Massaro \& Klitzke, 1977); (2) the letters of a word are recognized independently of each other; and (3) the contribution of a given letter to the recognizability of a word is proportional to its legibility. Table 1 illustrates a hypothetical example, where the probability of recognizing a directly fixated letter in a string of five letters is assumed to be 1 . Given the uniform drop in acuity characterized by the linear increase of the eyes' minimum angle of resolution over the central $10^{\circ}$ of the visual field (see, e.g., Olzak \& Thomas, 1986), we shall, for the sake of illustration, suppose that the probability of identifying neighboring letters in a string drops by a constant value-say, .03with each letter going rightward into peripheral vision. Thus, the probability of recognizing the letter immediately to the right of the fixated letter will be .97 ; for the following letters, it will be $.94, .91$, and so forth. Taking the aforementioned left/right asymmetry of letter legibility into account, the probability of recognizing the letter immediately to the left of fixation will drop by a value of $.03 * 1.8=.054$ with each letter of eccentricity. Thus, the probabilities of recognizing the letters to the left of the fixated letter will be $.946, .892, .838$, and so forth. The probability of recognizing the entire string can be estimated by multiplying the probabilities of recognizing the individual letters (see last column of Table 1 and the solid symbols in Figure 2). ${ }^{1}$ As can be seen from this example, the predicted probability of recognizing a five-letter string varies with fixation location in a way similar to that observed for word recognition.

Table 1

Theoretical Probability of Recognizing a Five-Letter String as a Function of Fixation Location

\begin{tabular}{|c|c|c|c|c|c|c|}
\hline \multirow{2}{*}{$\begin{array}{l}\text { Letter Position } \\
\text { Fixated in the } \\
\text { String }\end{array}$} & \multicolumn{5}{|c|}{ Position of Letter in the String } & \multirow{2}{*}{$\begin{array}{l}\text { Probability of } \\
\text { Recognizing the } \\
\text { Entire String }\end{array}$} \\
\hline & 1 & 2 & 3 & 4 & 5 & \\
\hline 1 & 1 & .97 & .94 & .91 & .88 & .73 \\
\hline 2 & .946 & 1 & .97 & .94 & .91 & .78 \\
\hline 3 & .892 & .946 & 1 & .97 & .94 & .77 \\
\hline 4 & .838 & .892 & .946 & 1 & .97 & .69 \\
\hline 5 & .784 & .838 & .892 & .946 & 1 & .55 \\
\hline
\end{tabular}

Note-Assumptions are: The probability of recognizing the directly fixated letter is equal to 1 . This probability drops linearly by .03 (arbitrary value) with each letter position of eccentricity to the right of fixation and by $1.8 \times .03=.054$ with each letter position of eccentricity to the left of fixation. See the text for details.

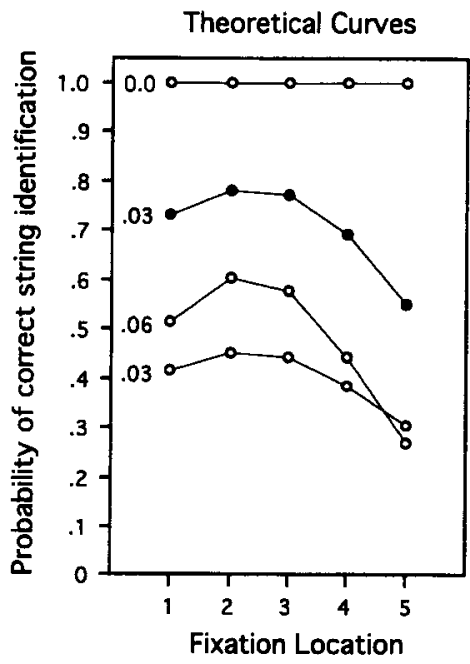

Figure 2. The theoretical probability of recognizing a five-letter string as a function of fixation location for four hypothetical legibility conditions. For the first three curves from the top, the probability of recognizing the letter at fixation point is 1 . As indicated in the panel, the drop-off rate of letter legibility with each letter of eccentricity to the right is 0 in the first, .03 in the second, and .06 in the third curve. For all curves, the drop-off rate to the left is 1.8 times the drop-off rate to the right. In the bottom curve the probability of recognizing the letter at the fixation point is .9 , and the drop-off rate to the right is .03 . The second curve from the top (solid symbols) represents the values calculated in the last column of Table 1.

\section{Evidence for a Relation Between the VPE and Letter Legibility}

Some supporting evidence for the assumed relation between the VPE and letter legibility comes from the observation that the same drop-off rate of letter legibility predicts the VPE for short and long words. For any fixation location and string length, theoretical probabilities of correct word recognition can be estimated by the equation

$$
\left.\begin{array}{l}
P_{\text {word }}\left(f, a, b_{\text {left }}, b_{\text {right }}, l\right)= \\
\left\{\left(\prod_{n=1}^{l-f}\left(a-n \cdot b_{\text {right }}\right)\right) \cdot a\right. \\
\left(\prod_{n=1}^{f-1}\left(a-n \cdot b_{\text {left }}\right)\right) \cdot a \cdot\left(\prod_{n=1}^{l-f} a-n \cdot b_{\text {right }}\right) \\
\left(\prod_{n=1}^{f-1}\left(a-n \cdot b_{\text {left }}\right)\right) \cdot a
\end{array}\right\} \begin{aligned}
& : f=1 \\
& 1<f<l, \\
& : f=l
\end{aligned}
$$

where $P_{\text {word }}\left(f, a, b_{\text {left }}, b_{\text {right }}, l\right)$ is the probability of recognizing a word as a function of $f$, the relative location of the fixated letter in the string (in letter units); $a$, the probability of recognizing the directly fixated letter; $b_{\text {left }}$ and $b_{\text {right }}$, the drop-off rate of the probability of recognizing a letter with increasing eccentricity, going left- and rightward; and $l$, the length of the word. 
With parameter $a=1$, we estimated parameter $b_{\text {left }}$ and $b_{\text {right }}$ from the empirical viewing position curve for five-letter words in Figure 1. To do so, the values of $b_{\text {left }}$ and $b_{\text {right }}$ were adjusted step by step until the shape of the theoretical viewing position curve matched the shape of the empirical curve for five-letter words (root mean square deviation [RMSD] between the theoretical and the empirical curve served as a goodness-of-fit measure). This was the case for a $b_{\text {left }}$ value of .057 and a $b_{\text {right }}$ value of .03 . (Note that the estimated ratio of the left/right asymmetry in the legibility of letter is $1.9 / 1$, which is very close to the ratio of $1.8 / 1$ obtained by Nazir et al., 1991.) Once fixed, the same values for the three parameters were used to calculate the theoretical curves for the remaining word lengths. The theoretical viewing position curves are presented, together with the empirical curves, in Figure 1 (open symbols). As can be seen from this comparison, although the height of the curves is systematically underestimated, the VPE (i.e., the shape of the curve) is captured by the model. To facilitate the comparison, we shifted the theoretical curves up to the height of the empirical curves (see gray curves in Figure 1) by adding a constant $h$ to Equation 1. Table 2 gives the value of $h$ for every word length, when RMSDs between the shifted theoretical curves and the empirical curves are minimal. The corresponding RMSDs are supplied in the table (see Appendix for calculations of RMSDs). Note that our model considers only visual factors. The discrepancy in height between the theoretical and the empirical curves - indicated by $h$-might therefore be explained by lexical factors. Support for this hypothesis comes from the observation that the heights of the word recognition curves change with word frequency (see, e.g., McConkie et al., 1989; O'Regan \& Jacobs, 1992; Vitu, 1991b).

However, although the strengthening of the VPE with increasing word length is captured by the model, the discrepancy between the empirical and the shifted theoretical curves becomes bigger as words become longer (see RMSD values in Table 2). This disparity is partly due to a floor effect. When string length increases, the theoretical curves drop considerably, and, as a consequence, the steepness of the branches of the curves becomes less pronounced. Thus, for a given drop-off rate, the theoretically predicted VPE might be more pronounced for short than for long words. However, as we will point out later, other factors contribute to the observed misfit.

Table 2

Value of the Constant $\boldsymbol{h}$ and Root Mean Square Deviations (RMSDs) Between the Empirical Data and the Shifted Theoretical Data in Figure 1

\begin{tabular}{llc}
\hline Word Length & $h$ & RMSD \\
\hline Five letters & .13 & .013 \\
Six letters & .19 & .027 \\
Seven letters & .28 & .030 \\
Eight letters & .33 & .069 \\
Nine letters & .39 & .048 \\
\hline
\end{tabular}

\section{A Test of the Model by Nazir et al. (1991)}

In the following study, we will test the assumption concerning the relation between letter legibility and the VPE by manipulating the legibility of letters in words. According to the model, manipulation of the legibility of letters in words will affect the shape of the word recognition curve in a systematic way. If, instead of .03 , as in Table 1 , parameter $b_{\text {right }}$ drops by a value of .06 per letter of eccentricity, indicating that the relative legibility of letters has decreased, the height of the word recognition curve is predicted to drop marginally and the VPE (i.e., the steepness of the branches of the curve) to be significantly strengthened. With a drop-off rate of 0 (this indicates that independently of their eccentricity, all letters of the word are identified equally well), the curve becomes flat. On the other hand, if the drop-off rate remains .03 but overall letter legibility - that is, parameter $a$-decreases (e.g., if, instead of 1 , the directly fixated letter is recognized only with a probability of .9), the VPE is predicted to be almost unaffected. But now overall performance drops considerably (see Figure 2 for these different examples).

Nazir et al. (1992) showed that the VPE indeed changes in the way predicted by the model. The authors manipulated the drop-off rate of letter legibility by varying the interletter spaces in words. Increasing the space between successive letters increases their eccentricity, which, in turn, decreases their legibility. The model thus predicts a stronger VPE for this case, and the prediction was confirmed by the results. As interletter space was progressively enlarged, the VPE became more and more pronounced. Other visual manipulations of the word stimuli that did not affect letter legibility (e.g., altering letter case) had no influence on the VPE but a considerable effect on the height of the curve. In the present study, the relative legibility of letters will be improved by magnifying the size of letters as they become further from fixation (Anstis, 1974). According to the model, the VPE should disappear when all letters of a word are perceived equally well across all eccentricities (i.e., when $b_{\text {left }} /$ $b_{\text {right }}=0$; see Figure 2).

\section{EXPERIMENT 1}

Psychophysical data have shown that peripheral vision, up to approximately $10^{\circ}$, can be considered to be functionally an expanded version of central vision, with a magnification factor of

$$
\mathbf{1}+m \Omega,
$$

where $\Omega$ is the eccentricity in degrees, and $m$ is a magnification parameter that varies between about 0.3 and 2 , depending on the type of acuity being measured (see, e.g., Goolkasian, 1994; Levi, Klein, \& Aitsebaomo, 1985; Olzak \& Thomas, 1986; O'Regan, 1989). In order to increase the relative legibility of letters, we magnified letter size in a way that was proportional to their distance from fixation, following Equation 2. Figure 3 gives schematic ex- 


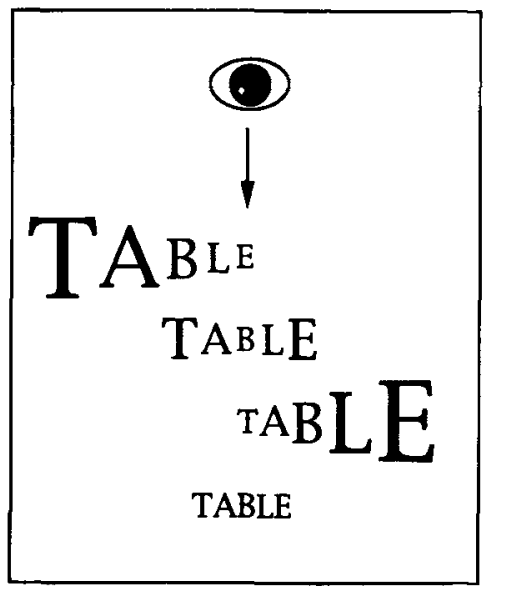

Figure 3. Schematic illustration of the butterfly words when fixated either at the end, at the middle, or at the beginning of the word, and the constant-sized small control word.

amples of the resulting word stimuli, which we refer to as butterfly words. Since we did not know the exact value of $m$ that was appropriate to the type of visual acuity involved in reading, we used three different $m$ values. According to the model, the drop-off rate of letter legibility decreases progressively with increasing $m$ values, and, as a consequence, the VPE for butterfly words should progressively diminish.

Note that the drop-off rate of letter legibility is being considered here as a parameter that can vary independently of the size of the letters. This is because, although the legibility of a single letter changes with size (e.g., A vs. A), a constant size change of all of the letters in a word does not strongly affect the visibility of the individual letters. For example, doubling the size of all the letters of a word makes them easier to see, but also doubles their eccentricity and so renders them less visible again (see O'Regan, 1989, 1990). This explains why, in the model, a constant decrease or increase of the size of all the letters of a word will mainly affect the height of the viewing position curve and only to a lesser extent its shape (see the second and the fourth graph from the top in Figure 2). Despite these arguments, to ensure that a flattening of the viewing position curve could not be attributed to the fact that letters in butterfly words are, on average, bigger than letters in words with constant-sized letters, in the work to be reported we included a control condition with constant-sized large letters. For these large words, we expected a VPE comparable to the one for words with constant-sized small letters.

As in the study by Nazir (1993), the subjects were asked to make a lexical decision to briefly presented letter strings.

\section{Method}

Subjects. Twenty-five undergraduate students from the University of Paris participated for partial course credit. All were native French speakers and had normal or corrected-to-normal vision.
Materials. Two hundred and fifty five-letter and nine-letter frequent French words (average frequency ranged from 27 to 198 per million, with a median of 57, for the five-letter words, and 28-211 per million and a median of 58 for the nine-letter words, selected from the Trésor de la langue Française, 1971) and 250 five-letter and nine-letter pronounceable pseudowords served as stimuli. The pseudowords were constructed by replacing one or two letters in words (other than the test words) having frequencies comparable to the test words. Words and pseudowords were displayed in capital letters. The stimuli were divided into five lists; each contained 50 words and 50 pseudowords of both lengths. Stimuli of each of the five lists were presented either with constant-sized small letters, with constant-sized large letters, or in one of the three butterfly versions, where letter size was scaled by a factor of $1+m \Omega$, with $m=1.1$, 1.3 , or 1.5. At a viewing distance of $100 \mathrm{~cm}$, the size of the letters in the constant-sized small words subtended a matrix of $0.15^{\circ}$ width and $0.17^{\circ}$ height; in the constant-sized large words, they subtended a matrix of $0.45^{\circ}$ width and $0.51^{\circ}$ height. For the butterfly words, the directly fixated letters had the size of the letters in the constant-sized small words. The small words are, therefore, the reference for the butterfly words. All stimuli were displayed in capital letters using a Times Roman vectorized font in white on dark on a Zenith video monitor at a $50 \mathrm{~Hz}$ refresh rate.

Design. The experiment contained five blocks. In each block, the stimuli of one of the five lists were presented either with constantsized small letters, with constant-sized large letters, or in one of the three butterfly versions. In each list, each word-length category was divided into five groups of 10 words/pseudowords, corresponding to the five different fixation zones. The attribution of a particular fixation zone to a particular word/pseudoword group was done differently for each of five groups of subjects, following a Latin Square design. Across all subjects, each word/pseudoword was seen an equal number of times from each fixation zone. Another Latin Square was used to attribute one of the five lists to one of the five presentation modes. Taken over all subjects, data were available for the eye fixating each of the five zones of each stimulus.

Procedure. Two vertically aligned bars appeared in the middle of the screen. The subjects were instructed to fixate the gap between the bars. Five hundred milliseconds after their onset, the bars were replaced by a letter string that was presented for $180 \mathrm{msec}$. No mask was used. The letter string was laterally displaced with respect to the fixation bars in such a way that, on its appearance, the subject's eye was positioned on one of the five letters in a five-letter string or on the first, third, fifth, seventh, or ninth letter of a nine-letter string. The subject's task was to decide as accurately as possible whether the string was a word or not by pressing one of two keys on the computer keyboard. The subjects used a chin rest.

\section{Results and Discussion}

Note that, in the following, we consider the results of correct lexical decisions to words only.

Five-letter words. Figure 4 (upper panel) summarizes the results of correct lexical decisions for five-letter words in the two control conditions (Figure 4a), and the three different magnification conditions (Figures $4 b, 4 c$, and 4d), together with a $95 \%$ confidence interval around the data points (Loftus \& Masson, 1994). The symbols represent empirical data, whereas the curves are theoretical data calculated with Equation 1. For this calculation, parameter $a$ was set to $a=1$. The values of parameters $b_{\text {left }}$ and $b_{\text {right }}$ were estimated from the empirical data of the five experimental conditions. The estimated values of parameter $b_{\text {right }}$, the ratio of $b_{\text {left }} / b_{\text {right }}$, the constant $h$ that is added to Equation 1 in order to match the heights of 

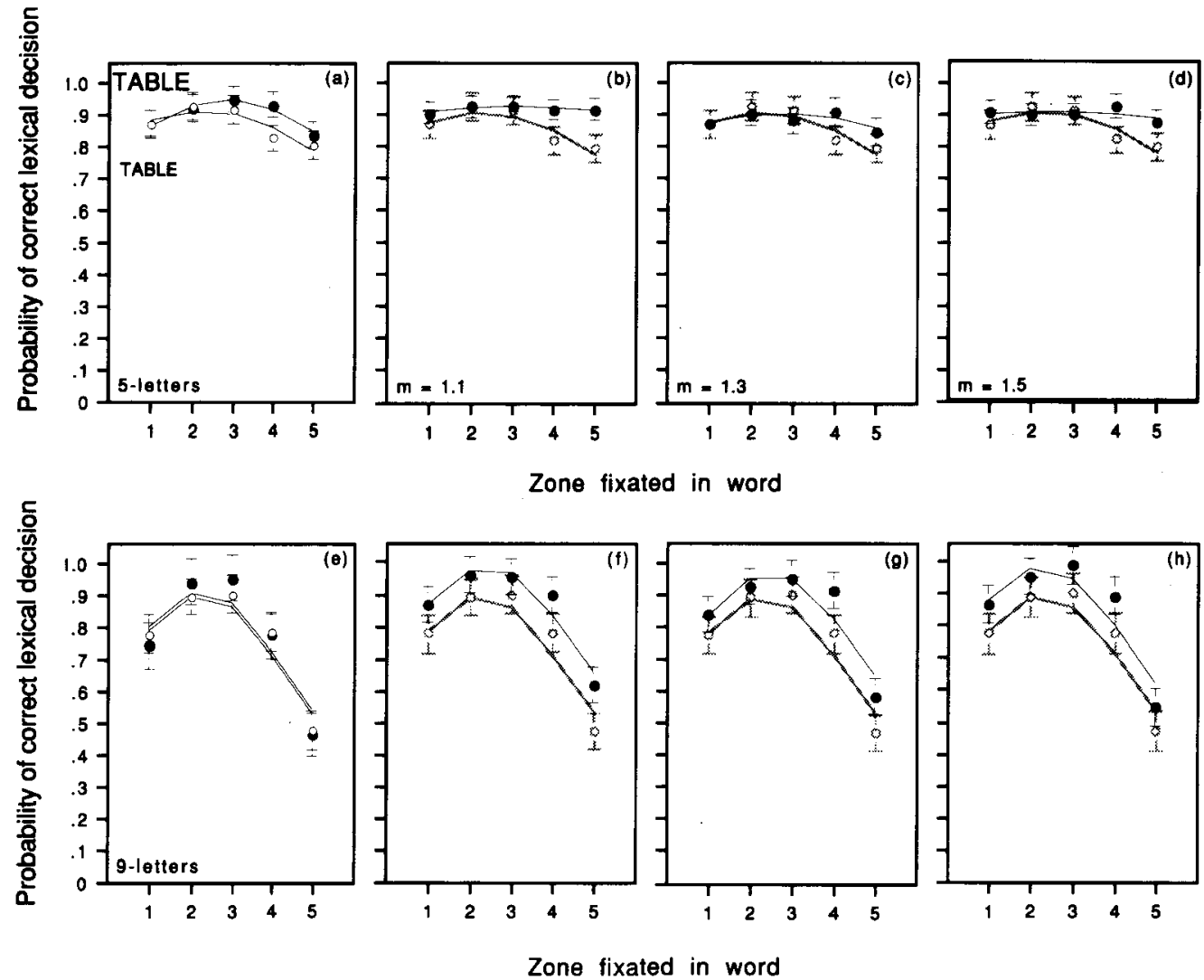

Zone fixated in word

Figure 4. The probability of correct lexical decision for five- and nine-letter words (Figures $4 \mathbf{a}-4 \mathrm{~d}$ and $4 \mathrm{e}-4 \mathrm{~h}$, respectively) as a function of fixation location in the different conditions of Experiment 1 . Note that the symbols represent empirical data, plotted with a $95 \%$ confidence interval, whereas the curves are theoretical data calculated with Equation 1 and shifted for a constant value $h$ (see text). Figures $4 \mathrm{a}$ and $4 \mathrm{e}$ represent performance for control words with constant-sized small (open symbols) and large (solid symbols) letters. The remaining graphs show performance for butterfly words (solid symbols), magnified either with $m=1.1, m=1.3$, or $m=1.5$. To make comparison easier, performance for the small control words (open gray symbols) is plotted together with performance for the butterfly words.

the theoretical and the empirical curves, and the corresponding RMSDs when the difference between the shifted theoretical curve and the empirical data is minimal are supplied in Table 3A. It is evident from Figure 4a that a VPE is obtained both for words with constantsized small letters and for words with constant-sized large letters. Except when fixating the fourth fixation zone, performance did not differ in the two conditions. Thus, increasing the size of all of the letters of a word does not affect the VPE. On the other hand, Figures $4 b, 4 c$, and $4 d$ indicate that, for butterfly words, the VPE disappears (see, as well, the corresponding values for parameter $b_{\text {right }}$ in Table 3A). This is already the case for the smallest $m$ value ( $m=1.1$ ); additional magnification ( $m=1.3$ and 1.5) does not further improve performance.

Nine-letter words. Figure 4 (lower panel) gives the corresponding results for nine-letter words, and Table 3B gives the estimates of parameter $b_{\text {right }}$ and the ratio of $b_{\text {left }} /$ $b_{\text {right. }}$. Figure $4 \mathrm{e}$ shows that performance for words with constant-sized small and large letters varies with the location of the eyes in the word; performance does not dif- fer between the two conditions. Figures $4 \mathrm{f}, 4 \mathrm{~g}$, and $4 \mathrm{~h}$ and the corresponding values of $b_{\text {right }}$ in Table 3B show that, contrary to five-letter words, magnifying letter size as a function of eccentricity does not alter the VPE for nine-letter words. Overall, performance for the butterfly words is slightly higher than for the constant-sized small words, but the shape of the curves does not change with letter magnification. As for five-letter words, no difference in performance is observed between the three $m$ values.

In short, compared with the small, nonbutterfly, reference words, an equal increase in the size of all of the letters in a word (constant-sized large words) has-as expected-almost no effect on performance. By contrast, magnifying letter size as a function of eccentricity (butterfly words), affects the shape of the viewing position curve when the word is short but its height when the word is long.

As will be shown in the following, the obtained pattern of results is indicative of an underestimation of the $m$ value. When $m$ is too small to equalize letter legibility across eccentricities, magnifying letter size will be less 
Table 3A

Estimated Values of Parameter $b_{\text {right }}$ of Equation 1, the Ratio of the Left/Right Asymmetry in Letter Legibility $\left(b_{\text {left }} / b_{\text {right }}\right)$, the Constant $h$, and Root Mean Square Deviations (RMSDs) Between the Shifted Theoretical Curves and the Empirical Curves of Five-Letter Words With Constant-Sized Small and Large Letters, and the Three Butterfly Words

\begin{tabular}{lcccc}
\hline Letter Size & $b_{\text {right }}$ & $b_{\text {left }} / b_{\text {right }}$ & $h$ & RMSD \\
\hline Small & .014 & $1.8 / 1$ & .01 & .020 \\
$m=1.1$ & .003 & $0.9 / 1$ & -.06 & .006 \\
$m=1.3$ & .009 & $1.2 / 1$ & -.04 & .015 \\
$m=1.5$ & .003 & $1.6 / 1$ & -.07 & .014 \\
Large & .025 & $1.1 / 1$ & .09 & .008 \\
\hline
\end{tabular}

Table 3B

Estimated Values of the Parameter, $b_{\text {right }}$ of Equation 1, the Ratio of Left/Right Asymmetry in Letter Legibility $\left(b_{\text {left }} / b_{\text {right }}\right)$, the Constant $h$, and Root Mean Square Deviations (RMSDs) Between the Shifted Theoretical Curves and the Empirical Curves of Nine-Letter Words With Constant-Sized Small and Large Letters and the Three Butterfly Words

\begin{tabular}{lcccc}
\hline Letter Size & $b_{\text {right }}$ & $b_{\text {left }} / b_{\text {right }}$ & $h$ & RMSD \\
\hline Small & .020 & $1.8 / 1$ & .31 & .046 \\
$m=1.1$ & .018 & $1.7 / 1$ & .36 & .036 \\
$m=1.3$ & .019 & $1.6 / 1$ & .35 & .055 \\
$m=1.5$ & .018 & $1.9 / 1$ & .37 & .060 \\
Large & .021 & $1.9 / 1$ & .35 & .064 \\
\hline
\end{tabular}

effective for short than for long words. Suppose that, in order to have equal legibility, the appropriate $m$ value is not $m=1.5$ but $m=1.7$. To equalize the legibility of a letter presented at an eccentricity of $0.5^{\circ}$ to a fixated letter of the size of $1^{\circ}$, the parafoveal letter has to be of the size of $1^{\circ} *\left(1+1.7 * 0.5^{\circ}\right)=1.85^{\circ}$ (see Equation 2$)$. With a magnification factor of $m=1.5$, the letter size is $1^{\circ} *$ $\left(1+1.5 * 0.5^{\circ}\right)=1.75^{\circ}$, which is only $1.75^{\circ} / 1.85^{\circ}=$ $95 \%$ of the hypothetically necessary size. For a letter presented at an eccentricity of $3^{\circ}$, letter size should be $1^{\circ} *$ $\left(1+1.7 * 3^{\circ}\right)=6.1^{\circ}$. However, with $m=1.5$, the letter size is $1^{\circ} *\left(1+1.5 * 3^{\circ}\right)=5.5^{\circ}$, which is now only $5.5^{\circ}$ $6.1^{\circ}=90 \%$ of the theoretically necessary size. In other words, if the magnification factor is underestimated, the discrepancy between the actual letter size and the size necessary to equalize legibility increases with increasing eccentricity. As is illustrated in Figure 5, our model predicts that even a small underestimation of the $m$ value would result in a different pattern of performance for the two word lengths. If, because of letter magnification, the drop-off rate of letter legibility decreases, for example, from .03 per letter position of eccentricity to .007 , the predicted curve for five-letter words is almost flat and only marginally above the reference curve. However, for nine-letter words, a pronounced VPE is still apparent, and the main effect of magnification is on the height of the curve.

\section{EXPERIMENT 2}

To verify whether the failure to flatten the word recognition curves for nine-letter words is caused by an underestimated $m$ value, we designed a new experiment in which the maximum $m$ value was increased to 1.7. In addition, we changed the smallest $m$ value to 0.5 and the intermediate $m$ value to 1.1, because the three $m$ values of Experiment 1 did not differ sufficiently in their effect on performance. Finally, we reduced presentation duration

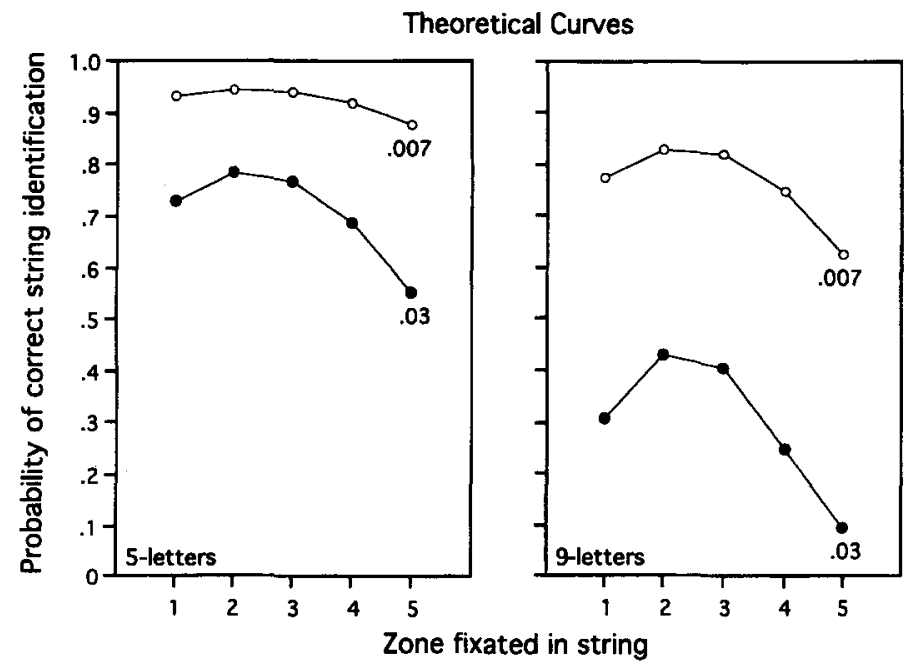

Figure 5. The theoretical probability of recognizing five- and nine-letter strings as a function of fixation location for two different drop-off rates of letter legibility, calculated with Equation 1 . For all curves, the probability of recognizing the letter at fixation is $a=1$. For the lower curves (solid symbols), the drop-off rate of letter legibility $b_{\text {right }}=.03$; for the upper curves (open symbols), $b_{\text {right }}=.007$. In both conditions, $b_{\text {left }}=1.8 * b_{\text {right }}$. 
from $180 \mathrm{msec}$ to $40 \mathrm{msec}$ in order to prevent a ceiling effect, which may have been present for the five-letter words in Experiment 1.

In Experiment 1, we had asked our subjects to make a lexical decision to the briefly presented stimuli. In order to generalize the results to other tasks, in Experiment 2 we additionally asked the subjects to name the word, once they had decided that the stimulus was a word.

\section{Method}

Subjects. Twenty-five undergraduate students from the University of Paris participated for partial course credit. All were native French speakers and had normal or corrected-to-normal vision. None of them had participated in Experiment 1.

Materials, Design, and Procedure. For technical reasons, in order to present nine-letter butterfly words magnified with an $m$ value of 1.7, the size of the reference letter at fixation had to be decreased. At a viewing distance of $100 \mathrm{~cm}$, the letters in the constant-sized small words (reference words) subtended a matrix of $0.13^{\circ}$ width and $0.16^{\circ}$ height; in the constant-sized large version, a letter subtended a matrix of $0.52^{\circ}$ width and $0.64^{\circ}$ height. The intermediate $m$ value was set to 1.1 , and the smallest to 0.5 . Presentation duration was $40 \mathrm{msec}$. As in Experiment 1, the subjects were asked to make a lexical decision. However, in order to minimize possible guessing effects, they were instructed to name the word once they had decided that the string was a word. The experimenter noted the answer, and only trials in which the word was named correctly were included in the analysis of the results. Apart from these changes, materials, design, and procedure were identical to those in Experiment 1 .

\section{Results and Discussion}

Note that we consider only the results for correctly named words.

Five-letter words. Figure 6 (upper panel) gives the results for correct naming of five-letter words, together with the $95 \%$ confidence intervals. As in the previous experiment, a VPE is obtained for words with constantsized small and large letters (see Figure 6a), although, in the present results, performance for large words is better than for small words. The difference in the height of these curves could be due to the absolute size of the letters in the small words. When letter size is too small and the probability of identifying the letter at fixation is less than 1 (i.e., parameter $a<1$ ), the model predicts a considerable drop of the word recognition curve and a slightly less pronounced effect of viewing position (see Figure 2
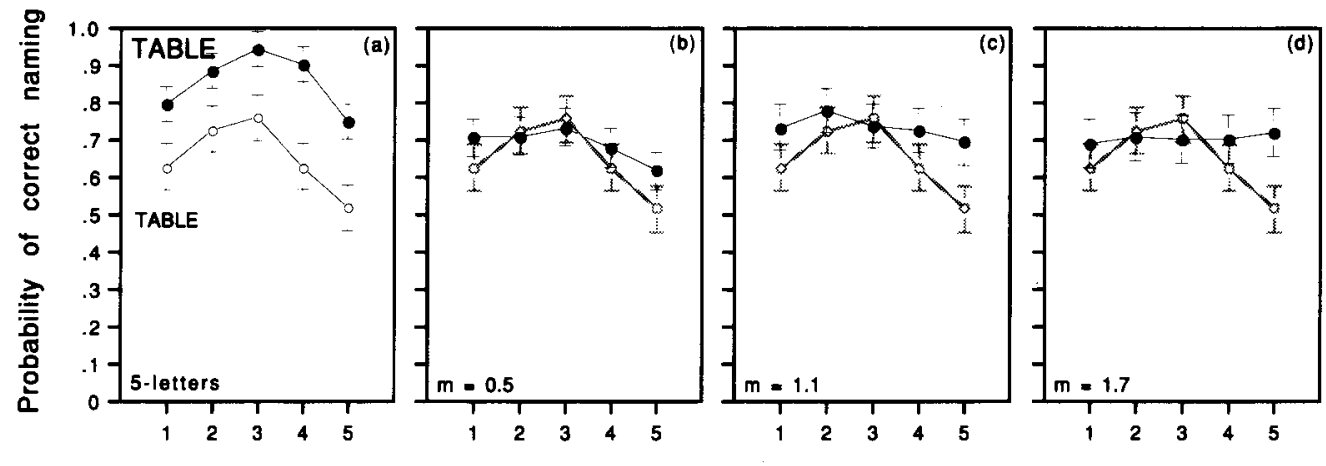

Zone fixated in word
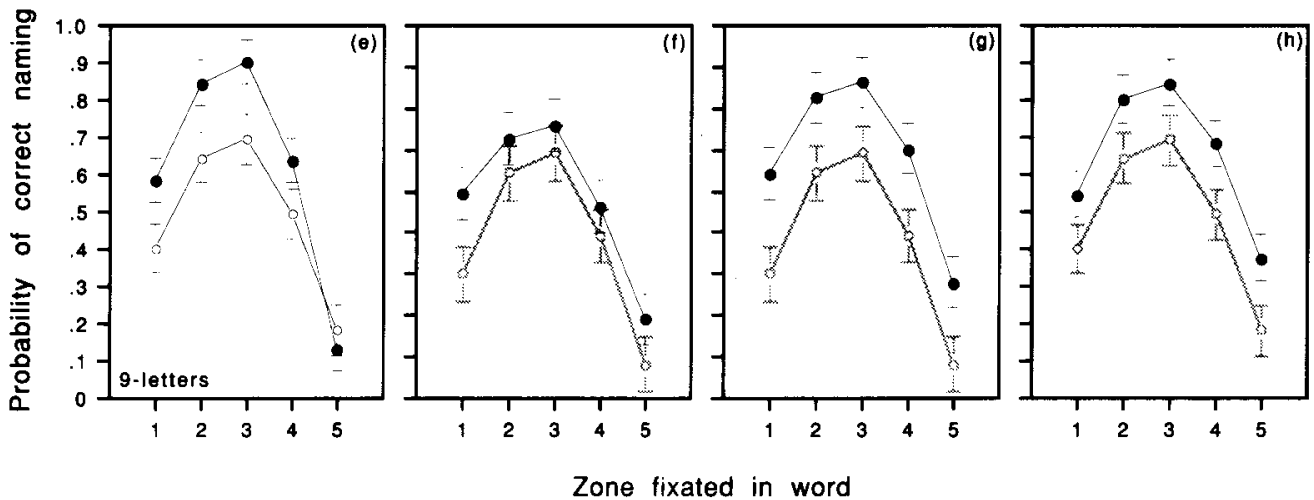

Zone fixated in word

Figure 6. The probability of the correct naming of five- and nine-letter words (Figures 6a-6d and 6e-6h, respectively) as a function of fixation location in the different conditions of Experiment 2. Data are plotted with a $95 \%$ confidence interval. No theoretical curves are presented in the figure. Figures $6 \mathrm{a}$ and $6 \mathrm{e}$ represent performance for control words with constant-sized small (open symbols) and large (solid symbols) letters. The remaining graphs show performance for the butterfly words (solid symbols), magnified either with $m=0.5, m=1.1$, or $m=1.7$. To make the comparison easier, performance for the small control words (open gray symbols) are plotted together with performance for the butterfly words. 
for this prediction). Experiment 3 will confirm this hypothesis. The probability of identifying the fixated letter is, in fact, below .75. Given this low value of parameter $a$, estimation of $b_{\text {left }}$ and $b_{\text {right }}$ becomes difficult, because, even for small drop-off rates, the predicted probabilities for recognizing a word tend toward zero. For this reason, we abandoned performing the estimations in this case. Thus, contrary to Figure 4, no theoretical curves are presented in Figure 6.

As in Experiment 1, magnifying letter size flattens the word recognition curves (see Figures $6 \mathrm{~b}, 6 \mathrm{c}$, and $6 \mathrm{~d}$ ). With an $m$ value of 0.5 , a weak viewing position effect is still apparent. This effect disappears, however, when the $m$ value is increased to 1.1 . A further increase of the $m$ value to 1.7 has no further effect on performance. Note that the height of all word recognition curves is lower in this experiment than in Experiment 1 . Since performance is highest for words with constant-sized large letters, and given that, for this condition, a VPE still exists, the flattening of the word recognition curves for the butterfly words cannot be attributed to a ceiling effect.

Nine-letter words. Figure 6 (lower panel) gives the corresponding results for nine-letter words. Figure 6e shows that performance for words with constant-sized small and large letters varies as a function of fixation location in the word. Performance for large words is better than for small words, and the VPE is slightly more pronounced in large words. As noted above, this is compatible with the assumption that the probability of identifying the directly fixated letter differs in the two conditions.

Figures $6 \mathrm{f}, 6 \mathrm{~g}$, and $6 \mathrm{~h}$ show that magnifying letter size as a function of eccentricity fails once more to flatten the viewing position curve for the long butterfly words. As in Experiment 1, magnifying letter size had an effect on the height but not on the shape of the viewing position curves. Compared with the small control words, overall performance increases slightly when letters are magnified with an $m$ value of 0.5 . A further increase is observed with an $m$ value of 1.1 , but, beyond this value, increasing letter size has no further effect on performance.

Basically, Experiment 2 replicates the results obtained in Experiment 1. Magnifying letter size as a function of eccentricity affects the shape of the word recognition curves when the words are short, but it affects the height when the words are long. Note that, in the present as well as in the previous experiment, letter magnification ceases to be effective after an $m$ value of 1.1 (this is indicated by the absence of a difference in performance for butterfly words magnified with $m$ values $1.1,1.3$, and 1.5 in Experiment 1 and $m$ values 1.1 and 1.7 in Experiment 2). It seems, thus, that the failure to flatten the word recognition curves for nine-letter words is not due to an underestimation of the $m$ value. Rather, we propose that it is due to an upper limit in the usefulness of the visual information made available by increasing letter size. We will come back to this point in the general discussion.

\section{EXPERIMENT 3}

In order to eliminate last doubts about possible contributions of visual factors in the failure to flatten the word recognition curves for nine-letter words, we designed an experiment in which subjects had to identify a target letter presented at various eccentricities in a string of five or nine Xs (e.g., XXXXXXAXX). The eye was fixating either the end or the beginning of the string, and letter size was magnified with eccentricity using the largest magnification factor of Experiment 2. If an $m$ value of 1.7 is still too small to equalize letter legibility across eccentricities, the probability of identifying the target letter should drop with an increasing distance of the target from fixation location.

\section{Method}

Subjects. Ten undergraduate students from the University of Paris participated for partial course credit. All were native French speakers and had normal or corrected-to-normal vision.

Materials. One hundred five-letter and 180 nine-letter strings of uppercase Xs served as stimuli. In each of the strings, one letter was replaced by 1 of 20 uppercase target letters. (All letters of the alphabet except the $\mathrm{X}$ itself and the letters I, J, L, T, and Q, served as targets. I, J, L, T, and Q were excluded from the set because a pilot study indicated that they were among the easiest targets to identify in the background of Xs.) The same target letter appeared once at each of the five and each of the nine letter positions in the two strings. The strings were presented either with constant-sized letters having the same size as the letters in the small words of Experiment 2 or with letters magnified with an $m$ value $=1.7$. The same font as that in Experiment 2 was used.

Procedure and Design. Presentation conditions were similar to those in Experiment 2. However, instead of five viewing conditions, in this experiment the string appeared on the screen such that the eye was either fixating the first or the last letter in the string. Thus, half of the time the target letter had to be identified in the right and half of the time in the left visual field. The experiment contained two blocks. In one of the blocks, the stimuli were presented with constantsized small letters; in the other block, the letters were magnified. Five- and nine-letter strings were presented together in one experimental block. The position of the critical letter in the string and the presentation side of the string (right or left of fixation) was randomized. Half of the subjects started with the constant-sized letter string condition, the other half with the magnified letter string condition. The task was to type the identified target letter on the computer keyboard. There was a short break halfway through the experiment.

\section{Results and Discussion}

Figure 7 gives letter identification performance as a function of target eccentricity and string length for letters presented in constant-sized letter strings (Figure 7a) and in strings with magnified letters (Figure $7 \mathrm{~b}$ ). The data are plotted, along with a $95 \%$ confidence interval, calculated separately for the two lengths and visual fields. Note that, because of reduced lateral masking, the typical increase in the probability of identifying the outer letters in the string is obtained (see, e.g., Nazir et al., 1991; Townsend et al., 1971). These values are excluded from the calculations of the confidence intervals. 


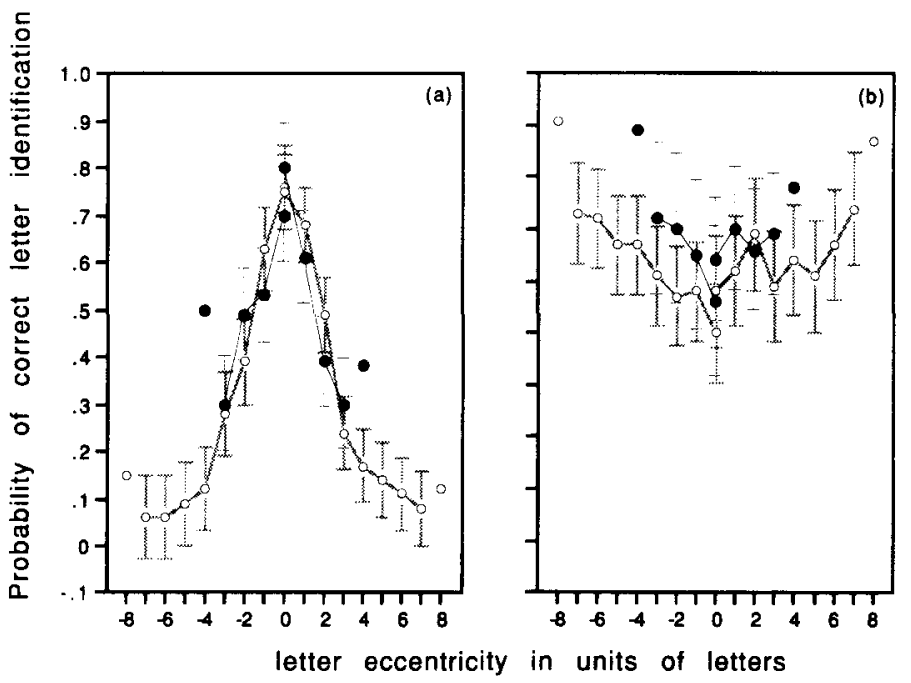

Figure 7. The probability of correct letter identification as a function of the distance of the target from fixation (in units of letters) when the target is presented in five- and nine-letter strings of Xs (solid and open symbols, respectively). Zero on the $x$-axis indicates fixation location; negative values indicate performance in the left visual field and positive values indicate performance in the right visual field. The data are plotted with a $95 \%$ confidence interval. Figure 7a shows performance for constant-sized small letters, Figure $7 b$ performance for magnified letters $(m=1.7)$.

As can be seen from Figure 7a, in constant-sized letter strings target identification drops rapidly with increasing eccentricity. Note that, at fixation location (eccentricity $0)$, the probability of identifying the target is below 1 . This confirms our hypothesis concerning the difference in the height of the word recognition curves for small and large constant-sized words in Experiment 2.

When letter size is magnified as a function of eccentricity, target letters are recognized equally well at all eccentricities (Figure $7 b$ ). This is true for both short and long letter strings. Thus, the results of Experiment 3 further corroborate the idea that the failure to flatten nine-letter words is not due to an underestimation of the $m$ value.

\section{GENERAL DISCUSSION}

Given the strong drop-off of letter legibility with eccentricity, any letter-based model of visual word recognition should predict that the probability of recognizing a word varies as a function of where the eyes fixate the word. The existence of a robust VPE during word recognition supports this assumption: A word is recognized best when the eye fixates the region around the center of a word, and performance decreases considerably when the word is fixated toward its beginning or end.

We used a simple model to allow easily testable quantitative predictions of the way the VPE effect should vary, if this phenomenon is related to letter legibility. Several features of the VPE could indeed be accounted for by this model. First, the fact that, for all word lengths, the optimal viewing position is systematically left of and not at word center is predicted by the model when the drop-off rate of letter legibility is assumed to be faster on the left of fixation than on the right (Nazir et al., 1991). Second, the strengthening of the VPE with word length emerges naturally as a consequence of the increasing number of letters that need to be processed. Third, a constant decrease or increase of the size of all of the letters does not affect performance, provided that (1) this manipulation does not change the drop-off rate of letter legibility, and (2) the probability of identifying the letter at fixation is not smaller than 1 . When this probability is smaller than 1 , the vertical offset of the viewing position curve decreases (Experiment 2 , for words with constant-sized letters). Fourth, when the drop-off rate of letter legibility is increased, the VPE becomes stronger (see Nazir et al., 1992).

All of these observations indicate the existence of a relation between the VPE and letter legibility. Yet, equalizing letter legibility across eccentricities does not entirely neutralize this effect. Increasing the size of letters as they come further from fixation does improve performance in the predicted way. However, beyond an $m$ value of 1.1, magnifying letter size ceases to be effective, and, as a consequence, although the VPE disappears for short words, it remains present for long words. Both the observation that magnifying letter size has no further effects beyond an $m$ value of 1.1 and the fact that an $m$ value of 1.7 was shown to be sufficient to equalize letter legibility across all eccentricities (Experiment 3 ) suggest that it is not the amount of visual information available, but rather the way this information is processed, that is the limiting factor here. From this point of view, improving visual conditions beyond a certain amount does not improve performance, because the information that is made 

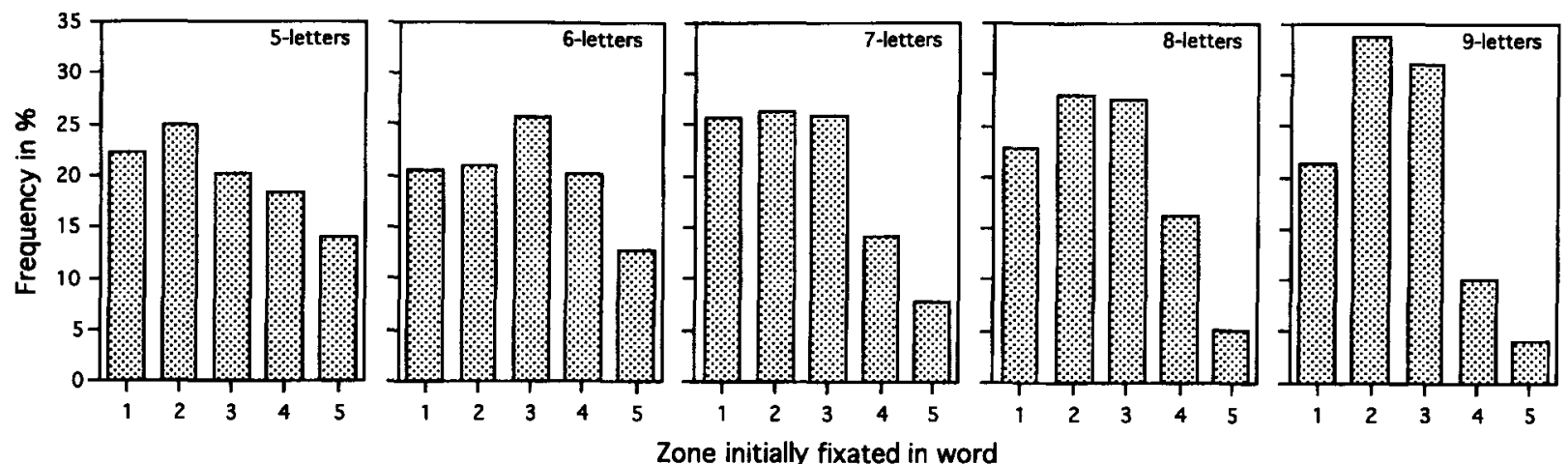

Figure 8. Frequency of initial landing position of the eye during reading, in words of five to nine letters. (Data replotted from Vitu et al., 1990.)

available by increasing letter size may not be part of the critical information that the system has learned to use to identify words during normal reading. ${ }^{2}$

More concretely, in order to quickly identify a word during reading, the system must process efficiently all visual information available from the word during a fixation. Because of the structure of the retina, visual cues available from a letter differ as a function of where it is presented with respect to the center of gaze (see, e.g., Bouma, 1971; Eriksen \& Schultz, 1979; Jacobs, Nazir, \& Heller, 1989). The closer a letter is to the fixation point, the more details there are that are available about it (i.e., high spatial frequency information). For parafoveally presented letters, mainly coarse, ambiguous features, such as the height-to-width quotient of the letter, can be extracted (i.e., low spatial frequency information). However, although information available from a parafoveally presented letter might be ambiguous as to the identity of a letter, the combined information from all of the letters in the word could be sufficient to allow correct identification of the word (Rumelhart \& Siple, 1974). In fact, "ambiguous" cues from parafoveal letters can become part of the set of critical features that the system is looking for in order to quickly (though not always perfectly) recognize a word. Thus, with reading experience, all visual cues available-including ambiguous features of parafoveal letters - become salient for the identity of the word. When details about parafoveal letters are made available artificially (e.g., by magnifying letter size), the infrequent use of these features in the context of a word will prevent them from being used as efficiently as the familiar "ambiguous" features. Therefore, in spite of their availability, these infrequent features, although potentially providing more information, will not contribute much to the identification of the word, and performance will remain unaffected by this manipulation.

That some kind of viewing-position-dependent perceptual learning takes place during reading is supported by the following observation. If we look at the landing site distribution of fixations in words during normal reading (see, e.g., McConkie, Kerr, Reddix, \& Zola, 1988; Rayner, 1979; Vitu et al., 1990; see Figure 8 for an ex- ample), there is a striking similarity between the distribution of these initial fixations in words and the word recognition curves in Figure 1: Word recognition performance varies with fixation location in the same way as the frequency of initial fixations. This apparent "preference" of the eye to land left of the center of a word is mainly determined by visuomotor factors and is not a strategy adopted by experienced readers in order to optimize information extraction (see, e.g., McConkie et al., 1988; Nazir, 1991; O'Regan, 1990; Vitu, 1991a; Vitu, O’Regan, Inhoff, \& Topolski, 1995). First-grade children locate their initial fixation on a word in much the same way that adult readers do (Grimes, 1989). It seems, thus, that performance is best at the location where the eyes tend to land most frequently.

Note that all of the arguments listed at the beginning of the General Discussion, including the compatibility between the location of the optimal viewing position and the left/right ratio of letter legibility, strongly suggest that word recognition is letter based. If a causal relation between the landing distribution and viewing-positiondependent word recognition performance really exists, it would therefore be misleading to interpret it as indicating that word recognition is based on some viewing-positiondependent global configuration of the visual stimulus. It seems, rather, that what is learned comprises the features of individual letters and where and how these features have been seen in the past.

\section{REFERENCES}

AdAms, M. J. (1979). Models of word recognition. Cognitive Psychology, 11, 133-176.

ANSTIS, S. M. (1974). A chart demonstrating variations in acuity with retinal position. Vision Research, 14, 589-592.

BoUMA, H. (1970). Interaction effects in parafoveal letter recognition Nature, 226, 177-178.

Bouma, H. (1971). Visual recognition of isolated lower case letters. $\mathrm{Vi}$ sion Research, 11, 459-474.

Bouma, H. (1973). Visual interference in the parafoveal recognition of initial and final letters of words. Vision Research, 13, 767-782.

Bouma, H., \& Legein, C. P. (1977). Foveal and parafoveal recognition of letters and words by dyslexics and by average readers. Neuropsychologia, 15, 69-80.

Brysbaert, M., \& D'Ydewalle, G. (1988). Callosal transmission in 
reading. In G. Luer, U. Lass, \& J. Shallo-Hoffmann (Eds.), Eye movement research: Physiological and psychological aspects (pp. 246266). Göttingen: Hogrefe.

Brysbaert, M., Vitu, F., \& Schroyens, W. (1996). The right visual field advantage and the optimal viewing position effect: On the relation between foveal and parafoveal word recognition. Neuropsychology, 10, 385-395.

ERIKSEN, C. W., \& Schultz, D. W. (1979). Temporal factors in visual information processing: A tutorial review. In J. Requin (Ed.), Attention and performance VII (pp. 3-23). Hillsdale, NJ: Erlbaum.

FARID, M., \& Grainger, J. (1996). How initial fixation position influences visual word recognition: A comparison of French and Arabic. Brain \& Language, 53, 351-368.

Goolkasian, P. (1994). Size scaling and its effect on letter detection. Perception \& Psychophysics, 56, 681-690.

GRIMES, M. (1989). Where first grade children look in words during reading. Unpublished master's thesis, University of Illinois, UrbanaChampaign.

Hagenzieker, M. P., van der Heijden, A. H. C., \& Hagenaar, R. (1990). The time courses in visual-information processing: Some empirical evidence for inhibition. Psychological Research, 52, 13-21.

Holmes, V., \& O'Regan, J. K. (1987). Decomposing French words. In J. K. O'Regan \& A. Lévy-Schoen (Eds.), Eye movements: From physiology to cognition (pp. 459-466). Amsterdam: North-Holland.

JaCoBs, A. M., Nazir, T. A., \& Heller, O. (1989). Perception of lowercase letters in peripheral vision: A discrimination matrix based on saccade latencies. Perception \& Psychophysics, 46, 95-102.

Levi, D. M., Klein, S. A., \& AitsebaOmo, A. P. (1985). Vernier acuity, crowding and cortical magnification. Vision Research, 25, 963-977.

LoFtus, G. R., \& Masson, M. E. J. (1994). Using confidence intervals in within-subject designs. Psychonomic Bulletin \& Review, 1, 476-490.

MASSARO, D. W., \& KLITZKE, D. (1977). Letters are functional in word identification. Memory \& Cognition, 5, 292-298.

McConkie, G. W., KerR, P. W., Reddix, M. D., \& Zola, D. (1988). Eye movement control during reading: I. The location of initial eye fixations in words. Vision Research, 28, 1107-1118.

McConkie, G. W., Kerr, P. W., Reddix, M. D., Zola, D., \& JacobS, A. M. (1989). Eye movement control during reading: II. Frequency of refixating a word. Perception \& Psychophysics, 46, 245-253.

NAZIR, T. A. (1991). On the role of refixations in letter strings: The influence of oculomotor factors. Perception \& Psychophysics, 49, 373-389.

NAzIR, T. A. (1993). On the relation between the optimal and the preferred viewing position in words during reading. In G. d'Ydewalle \& J. van Rensbergen (Eds.), Perception and cognition: Advances in eye movement research (pp. 349-361). Amsterdam: North-Holland.

Nazir, T. A., Heller, D., \& Sussmann, C. (1992). Letter visibility and word recognition: The optimal viewing position in printed words. Perception \& Psychophysics, 52, 315-328.

NAZIR, T. A., O'REGAN, J. K., \& JACOBS, A. M. (1991). On words and their letters. Bulletin of the Psychonomic Society, 29, 171-174.

OlzaK, L. A., \& Thomas, J. P. (1986). Seeing spatial patterns. In K. R. Boff, L. Kaufman, \& J. P. Thomas (Eds.), Handbook of perception and human performance (Vol. 2, pp. 7:1-7:56). New York: Wiley.

O'REGAN, J. K. (1989). Visual acuity, lexical structure, and eye movements in word recognition. In B. Elsendoorn \& H. Bouma (Eds.), Models of human perception (pp. 261-292). London: Academic Press.

O'Regan, J. K. (1990). Eye movements and reading. In E. Kowler (Ed.), Eye movements and their role in visual and cognitive processes (pp. 395-453). Amsterdam: Elsevier.

O'REGAN, J. K., \& JACOBS, A. M. (1992). Optimal viewing position effect in word recognition: A challenge to current theory. Journal of Experimental Psychology: Human Perception \& Performance, 18, 185-197.

O'Regan, J. K., Lévy-Schoen, A., Pynte, J., \& Brugaillère, B. (1984). Convenient fixation location within isolated words of different length and structure. Journal of Experimental Psychology: Human Perception \& Performance, 10, 250-257.

RADACH, R., \& KeMPE, V. (1993). An individual analysis of initial fixation position in reading. In G. d'Ydewalle \& J. van Rensbergen (Eds.), Perception \& cognition: Advances in eye movement research (pp. 213-225). Amsterdam: North-Holland.
RAYNER, K. (1979). Eye guidance in reading: Fixation location within words. Perception, 8, 21-30.

Rayner, K., \& PollatSeK, A. (1989). The psychology of reading. Englewood Cliffs, NJ: Prentice-Hall.

Rumelhart, D. E., \& SiPle, P. (1974). The process of recognizing tachistoscopically presented words. Psychological Review, 81, 99-118.

ToWnSEND, J. T., TAYLOR, S. G., \& BROWN, D. R. (1971). Lateral masking for letters with unlimited viewing time. Perception \& Psychophysics, 10, 375-378.

Trésor de la Langue Française [French language frequency counts]. (1971). Nancy: Centre National de la Recherche Scientifique.

Underwood, G., Clews, S., \& Everatt, J. (1990). How do readers know where to look next? Local information distributions influence eye fixations. Quarterly Journal of Experimental Psychology, 42A, 39-65.

VITU, F. (1991a). The existence of a center of gravity effect during reading. Vision Research, 31, 1289-1313.

VITU, F. (1991b). The influence of parafoveal preprocessing and linguistic context on the optimal landing position effect. Perception \& Psychophysics, 50, 58-75.

Vitu, F., O'Regan, J. K., InHOFF, A. W., \& Topolski, R. (1995). Mindless reading: Eye-movement characteristics are similar in scanning letter strings and reading texts. Perception \& Psychophysics, 57, 352-364.

Vitu, F., O'Regan, J. K., \& MitTaU, M. (1990). Optimal landing position in reading isolated words and continuous text. Perception \& Psychophysics, 47, 583-600.

\section{NOTES}

1. Adams (1979) showed that the probabilities of recognizing letters in words are not independent, in the sense that letter clusters can be recognized together. It is partly because of this dependency (orthographic structure) that we can recognize words without having seen all letters. In the frame of our model, orthographic structure could be implemented as the following. Instead of a linear drop of letter recognition probabilities with eccentricity, recognition probabilities could drop, for example, in the following way: $1, .97, .94, .94, .88$, when fixated at the first letter; $.946,1, .97, .97, .91$, when fixated at the second; $.892, .946,1,1$, .94 , the third; $.838, .892,1,1, .97$, the fourth; and $.784, .838, .946, .946$, 1 , the fifth letter. With this letter cluster, the theoretical probabilities of recognizing the whole string from the five viewing positions would be $.75, .81, .79, .73$, and .59 (instead of $.73, .78, .77, .69$, and .55 , as in the last column of Table 1). As can be seen from these calculations, unless the letter cluster is very large, orthographic structure does not appreciably change the VPE itself. For mathematical tractability, we will work with the independence assumption.

2. We think that the failure to flatten the word recognition curves for nine-letter words cannot be attributed to lexical factors. Any viewingposition-dependent variation of word recognition performance that is related to lexical factors must be mediated by the legibility of letters. Lexical characteristics of a word do not change with fixation location. What changes is the availability of information as a function of where the eye fixates with respect to the lexically informative part of the word. By equalizing letter legibility across eccentricities, disadvantages because of nonoptimal fixation locations are compensated, and, therefore, the VPE should disappear.

\section{APPENDIX}

Root mean square deviations (RMSDs) were calculated with the equation

$$
R M S E=\sqrt{\sum \frac{\left(O_{i}-P_{i}\right)^{2}}{n}},
$$

where $P_{i}$ is the predicted value and $O_{i}$ is the observed value, averaged over all subjects.

(Manuscript received July 8, 1996 revision accepted for publication March 20, 1997.) 